\title{
Possible anti-inflammatory effect of salmeterol against interleukin-8 and neutrophil activation in asthma in vivo
}

\author{
D.W. Reid*, C. Ward", N. Wang*, L. Zheng*, R. Bish*, B. Orsida*, E.H. Walters
}

Possible anti-inflammatory effect of salmeterol against interleukin-8 and neutrophil activation in asthma in vivo. D.W. Reid, C. Ward, N. Wang, L. Zheng, R. Bish, B. Orsida, E.H. Walters. (C) ERS Journals Ltd 2003.

ABSTRACT: In-vitro data suggest that long-acting $\beta_{2}$-agonists may have a neutrophilstabilising effect. A reduction in airway wall eosinophil number following introduction of salmeterol in persistent asthma has previously been described. There is currently interest in the "neutrophil system" in asthma, and thus the aim of the present study was to investigate the effect of salmeterol on interleukin (IL)-8, neutrophils and myeloperoxidase (MPO) in persistent asthma.

In the same 12-week double-blind parallel-group placebo-controlled study as described previously, the effects on bronchoalveolar lavage fluid (BALF) IL-8, neutrophils and MPO of introducing salmeterol $(50 \mu \mathrm{g}$ b.i.d.) or giving additional inhaled corticosteroid (fluticasone $100 \mu \mathrm{g}$ b.i.d.) in 45 subjects with persistent asthma already on low/moderate doses of inhaled corticosteroids were further investigated.

At baseline, BALF IL-8 but not neutrophil or MPO levels were significantly raised in the asthmatic subjects compared to normal controls. MPO levels correlated strongly with IL-8 levels, and weakly with BALF neutrophil numbers in the asthmatics. Fluticasone treatment resulted in significantly elevated neutrophil numbers, but not MPO or IL-8 levels. In contrast, introducing salmeterol significantly reduced IL-8 and MPO levels, but did not affect BALF neutrophil numbers. Interestingly, salmeterol and fluticasone showed significantly contrasting effects on MPO and neutrophils, and there was a divergent effect on IL-8 levels that almost reached significance.

Excessive interleukin-8 levels may be relevant to asthma pathogenesis, even in the setting of moderate-dose inhaled corticosteroid therapy. Reduction in interleukin-8 production and possibly stabilisation of airway neutrophil numbers may explain the greater clinical benefit of adding a long-acting $\beta_{2}$-agonist rather than merely increasing inhaled corticosteroid doses. Indeed, high-dose inhaled corticosteroid therapy alone may promote airway neutrophilia.

Eur Respir J 2003; 21: 994-999.
*Dept of Respiratory Medicine, the Alfred Hospital and Monash University Medical School, Melbourne and "Discipline of Medicine, University of Tasmania Medical School, Hobart, Australia. ${ }^{*}$ Lung Biology and Transplant Group, University of Newcastle upon Tyne and the Freeman Hospital, Newcastle upon Tyne, UK.

Correspondence: E.H. Walters, University of Tasmania Medical School, Hobart, 7001, Tasmania, Australia.

Fax: 61362264894

E-mail: Haydn.Walters@utas.edu.au

Keywords: Asthma

bronchoalveolar lavage

interleukin-8

myeloperoxidase

neutrophils

salmeterol

Received: November 272002

Accepted after revision: January 232003

This study was supported by the National Health and Medical Research Council, (Canberra, Australia), Alfred Hospital Foundation (Melbourne, Australia), Royal Hobart Hospital Research Foundation (Hobart, Australia) and GlaxoSmithKline (Boronia, Australia).
Asthma is characterised by airway inflammation, bronchial hyperresponsiveness and reversible airflow obstruction. The asthmatic inflammatory response consists of infiltration into the airway of a variety of activated inflammatory cells with release of mediators of asthma pathophysiology. The eosinophil has an established role as an effector cell in asthma but the pro-inflammatory role of other granulocytes, in particular the neutrophil, remains less well defined $[1,2]$.

Increased numbers of airway neutrophils have been described in severe steroid-dependent asthma, during acute exacerbations and in cases of sudden-onset fatal disease [3-5]. An increase in neutrophil activation, but not numbers, in mild chronic asthma has previously been shown [6]. The role of airway neutrophils in the pathogenesis of persistent asthma despite mild/moderate inhaled corticosteroid (ICS) doses has not been established. However, more recent data confirm considerable heterogeneity of the asthma phenotype and many subjects may exhibit an airway disease characterised by sputum neutrophilia rather than an eosinophilic inflammatory pattern [7-9].

In-vitro data suggest that long-acting $\beta_{2}$-agonists (LABAs) may have a neutrophil modulatory effect and such an effect could explain the reductions in asthma exacerbations seen with LABAs [10]. A significant decrease in activated eosinophil numbers in ICS-treated, but still symptomatic, asthmatic subjects following introduction of salmeterol xinafoate has previously been described [11]. In the current report, based on the same asthmatic individuals, assessment of whether neutrophils or neutrophil activation may be relevant to disease or treatment effects in these individuals was carried out by determining the concentrations of the neutrophilrelated chemokine interleukin (IL)- 8 and activation marker myeloperoxidase (MPO) in bronchoalveolar fluid (BALF) at baseline and with either increased ICS dose or supplementary LABA treatment.

\section{Methods}

\section{Subjects}

Fifty subjects were initially recruited from the asthma volunteer database of the Dept of Respiratory Medicine (Alfred Hospital and Monash University Medical School, Melbourne, Australia) to undergo baseline assessment including bronchoscopy with bronchoalveolar lavage (see [11] for full details of recruitment and screening) (table 1). All 
Table 1.-Asthma patient baseline characteristics by treatment group

\begin{tabular}{|c|c|c|c|}
\hline & Placebo & Salmeterol & Fluticasone \\
\hline Subjects n & 16 & 13 & 16 \\
\hline Males/females n & $7 / 9$ & $8 / 5$ & $11 / 5$ \\
\hline Age yrs & $33(22-68)$ & $38(20-70)$ & $42(22-63)$ \\
\hline ICS dose $\mu \mathrm{g} \cdot$ day $^{-1}$ & $400(200-500)$ & $400(200-500)$ & $400(200-500)$ \\
\hline Atopic/nonatopic $n$ & $12 / 4$ & $13 / 0$ & $11 / 5$ \\
\hline PD20 mg & $0.011(0.001-0.16)$ & $0.022(0.01-0.56)$ & $0.021(0.003-0.46)$ \\
\hline FEV1 \% pred & $83(61-109)$ & $84(63-106)$ & $80(61-102)$ \\
\hline 24-h symptom score & $1.07(0.57-4.21)$ & $1.21(0.01-3.29)$ & $1.39(0.57-2.57)$ \\
\hline $\begin{array}{l}\text { 24-h salbutamol use } \\
\text { puffs } \cdot \text { day }^{-1}\end{array}$ & $1.07(0.57-5.50)$ & $1.21(0.57-4.86)$ & $1.82(0.50-5.36)$ \\
\hline
\end{tabular}

Data are presented as median (range), and as geometric mean (range) for provocative dose of methacholine causing a $20 \%$ fall in forced expiratory volume in one second (FEV1) (PD20). Full clinical details are presented in [14]. ICS: inhaled corticosteroid; \% pred: percentage of the predicted value.

subjects had a history of asthma of $\geqslant 12$ months' duration and all were nonsmokers aged $20-70$ yrs. All subjects had received treatment with up to $500 \mu \mathrm{g} \mathrm{ICS} \cdot \mathrm{day}^{-1}$ (range $100-500 \mu \mathrm{g} \mathrm{ICS} \cdot \mathrm{day}^{-1}$ ) for a period of $\geqslant 1 \mathrm{yr}$. All subjects were required to be "actively asthmatic", i.e. they had to satisfy at least one of the following criteria: 1) a symptom score of $>2$ on seven of the preceding 14 days; 2 ) the need for rescue medication on more than seven of the preceding 14 days; and 3) peak expiratory flow variability of $<15 \%$ on at least seven of the last 14 days, as well as some degree of symptoms and rescue medication use over the same period. At baseline, all subjects had a forced expiratory volume in one second (FEV1) of $\geqslant 60 \%$ of the predicted value, and subjects who had a history of a recent (within the last 4 weeks) hospital admission with an exacerbation of asthma or upper or lower respiratory tract infection or recent change in medication were excluded. Thirteen nonsmoking nonatopic individuals were recruited as normal controls. The study was approved by the Alfred Hospital Ethics Committee and all subjects gave written informed consent prior to entry.

\section{Study design}

Baseline bronchoscopy was performed after a 2-6-week run-in period, during which asthmatic subjects continued taking their normal ICS dose plus salbutamol $200 \mu \mathrm{g}$ as required via a metered-dose inhaler. Subjects who met the inclusion criteria of needing to be currently symptomatic underwent bronchoscopy and were then randomised to the intervention study which was double-blind, randomised, placebo-controlled and parallel-group in design. This was the same study as described previously [11]. During the 12-week treatment period, subjects received either salmeterol $50 \mu \mathrm{g}$ b.i.d., fluticasone $100 \mu \mathrm{g}$ b.i.d. (approximately doubling the median daily ICS dose) or identical placebo, all given as a dry powder via a Diskhaler (GlaxoSmithKline, Uxbridge, UK). During the study, subjects continued to take their usual regular ICS dose and salbutamol as required. Randomisation was performed within the hospital pharmacy using a computer-generated list of random numbers and medications were dispensed in identical devices. Bronchoscopy in asthmatic subjects was repeated after 12 weeks of study treatment. The 13 normal controls underwent bronchoscopy only once to obtain comparative data.

\section{Fibreoptic bronchoscopy and bronchoalveolar lavage fluid cell processing}

Full details of the fibreoptic bronchoscopic procedure and processing of BALF have been given previously [11]. Briefly, subjects were sedated with intravenous midazolam and topical lidocaine ( $4 \%$ above the vocal cords, $2 \%$ below) was used to anaesthetise the airways. Bronchoalveolar lavage was performed to yield three $60-\mathrm{mL}$ aliquots from the right middle lobe using a standard protocol. Total cell counts were determined on unfiltered BALF using a Neubauer haemocytometer and cytocentrifuge preparations were stained with

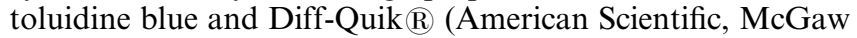
Park, IL, USA) to allow identification and determination of mast cells and BALF absolute and differential cell counts, respectively.

\section{Enzyme-linked immunosorbent assays}

Bronchoalveolar lavage fluid interleukin-8. IL-8 levels were measured in unconcentrated cell-free BALF using a commercially available enzyme-linked immunosorbent assay (ELISA) kit with precoated microtitre plates (Amersham International plc, Little Chalfont, UK). The detection range was 10$1,000 \mathrm{pg} \cdot \mathrm{mL}^{-1}$. Fifty microlitres of standard and BALF were incubated in duplicate. After washing away any unbound sample proteins, a biotinylated antibody directed against IL-8 was added to the wells, which were then washed again and incubated with antistreptavidin conjugated to horseradish peroxidase. After adding the substrate, colour developed in proportion to the amount of IL- 8 bound in the initial step. The absorbance was measured in an ELISA reader (Model 450; Bio-Rad, Hercules, CA, USA) at a wavelength of $450 \mathrm{~nm}$. The amount of IL-8 present in the samples was calculated by reference to the wells containing known dilutions of standard.

Bronchoalveolar lavage fluid myeloperoxidase. BALF MPO levels were also measured in unconcentrated fluid in an essentially identical method to that used for IL-8, utilising a monoclonal anti-MPO antibody in a commercially available ELISA kit (Bioxytech, Oxis International, Portland, OR, USA).

\section{Statistical analysis}

Baseline data were not normally distributed. Logarithmic transformation normalised BALF neutrophil numbers and MPO levels and these data were therefore expressed as geometric mean and 95\% confidence interval (CI). IL-8 concentrations remained non-normally distributed following logarithmic transformation. Differences between asthmatic and normal subjects at baseline were therefore compared using either unpaired t-tests or the Mann-Whitney U-test, as appropriate for the distribution of the data. Relationships between neutrophil numbers and IL- 8 and MPO levels were 
tested using Pearson's correlation coefficient. In order to adjust for baseline values, two-way analysis of covariance (ANCOVA) was used for the intervention study. In cases in which ANCOVA identified significant differences, unpaired t-tests were used to test for differences between individual treatments. Treatment differences for nonparametrically distributed data (IL-8) were assessed using the Kruskal-Wallis test. Additionally, changes compared to baseline within the group or groups implicated were analysed using either paired t-tests or Wilcoxon signed-rank tests. BALF cell differentials were analysed as both percentages and absolute counts, but the results were similar irrespective of how the data were presented. Relationships between the relative changes with treatment were analysed using logarithmically transformed data and Pearson's correlation coefficient. A two-tailed $\mathrm{p}<0.05$ was considered significant.

\section{Results}

The present analysis is based on data from the 45 asthmatic subjects who underwent baseline bronchoscopy and completed the 12-week intervention study. Of the five subjects who withdrew after randomisation, two decided not to continue after the first bronchoscopic procedure and three developed asthma exacerbations (one in the placebo group and two in the salmeterol group). Full details of clinical outcomes have been given previously [11]. However, both fluticasone and salmeterol significantly improved clinical indices compared to placebo, and these changes were most marked in the salmeterol group. The provocative dose of methacholine causing a 20\% fall in FEV1 (PD20) improved significantly and to a similar degree in both the fluticasone and salmeterol groups.

\section{Asthmatic subjects versus normal controls}

BALF IL-8 levels were significantly elevated at baseline in the asthmatic subjects (median (range) 67.7 (16.1-540.4) $\left.\mathrm{pg} \cdot \mathrm{mL}^{-1}\right)$ compared to normal controls $\left(26.1(8.7-38.7) \mathrm{pg} \cdot \mathrm{mL}^{-1}\right.$, $\mathrm{p}=0.001$ ).

There was no significant difference between asthmatic subjects and normal controls in BALF absolute neutrophil counts (geometric mean $(95 \% \mathrm{CI}) 2.1 \times 10^{3}\left(1.5 \times 10^{3}-2.8 \times 10^{3}\right)$ versus $2.9 \times 10^{3}\left(2.1 \times 10^{3}-3.9 \times 10^{3}\right)$ cells $\left.\cdot \mathrm{mL}^{-1}, \mathrm{p}=0.3\right)$, or MPO levels (3.0 (2.1-4.2) versus $\left.1.6(0.6-3.9) \mathrm{ng} \cdot \mathrm{mL}^{-1}, \mathrm{p}=0.5\right)$.

\section{Relationships at baseline}

At baseline, there was a strong relationship between BALF IL- 8 and MPO levels $\left(\mathrm{r}^{2}=0.6, \mathrm{p}<0.0001\right)$ amongst the asthmatics. Similarly, there was a weaker but significant relationship between MPO levels and BALF neutrophil numbers $\left(\mathrm{r}^{2}=0.19\right.$, $\mathrm{p}=0.003)$ but not BALF eosinophil numbers $\left(\mathrm{r}^{2}=0.01, \mathrm{p}=0.5\right)$. There was no significant relationship between IL-8 levels and BALF neutrophil numbers $\left(r^{2}=0.1, p=0.08\right)$ or eosinophil numbers $\left(\mathrm{r}^{2}=-0.003, \mathrm{p}=0.7\right)$. There was no relationship between either percentage or absolute eosinophil and neutrophil numbers.

Log PD20 was significantly, but weakly and negatively, related to both BALF neutrophil numbers $\left(r^{2}=0.1, p=0.03\right)$ and MPO levels $\left(\mathrm{r}^{2}=0.1, \mathrm{p}=0.04\right)$, but no relationship with IL-8 levels or BALF eosinophil or epithelial cell numbers was seen.

\section{Drug effects}

Bronchoalveolar lavage fluid neutrophil numbers and cell differentials. The two-way ANCOVA for logarithmically transformed data indicated significantly contrasting effects of salmeterol and fluticasone on BALF neutrophils $(\mathrm{p}=0.007)$, with placebo having no effect (table 2, fig. 1).

Direct within-group comparisons confirmed that giving additional ICS in the form of fluticasone resulted in significantly elevated total neutrophil numbers, from a geometric mean of $2.2 \times 10^{4}$ to $4.6 \times 10^{4}$ cells $\cdot \mathrm{mL}^{-1} \quad(\mathrm{p}=0.001)$, but this was not significant compared to placebo $(\mathrm{p}=0.2)$. The slight reduction in mean neutrophil numbers after treatment with salmeterol, from $2.2 \times 10^{4}$ to $1.7 \times 10^{4}$ cells $\cdot \mathrm{mL}^{-1}$, was not significant $(\mathrm{p}=0.3)$.

Bronchoalveolar lavage fluid interleukin-8. Analysis of withintreatment group changes revealed a significant reduction in IL-8 levels with introduction of salmeterol $(\mathrm{p}=0.02)$, but no treatment effect for fluticasone $(\mathrm{p}=0.6)$ (table 2, fig. 2). The Kruskal-Wallis test revealed contrasting effects upon IL-8 levels for salmeterol and fluticasone that reached only borderline significance $(\mathrm{p}=0.06)$, but there was no significant difference between salmeterol and placebo $(\mathrm{p}=0.09)$.

Bronchoalveolar lavage fluid myeloperoxidase. ANCOVA also revealed significant and contrasting treatment differences between salmeterol and fluticasone on MPO levels $(\mathrm{p}=0.002)$ (table 2, fig. 3). The reduction in MPO levels with salmeterol was also significant compared to placebo $(\mathrm{p}=0.04)$. Similarly, the within-group reduction in geometric mean MPO levels following treatment with salmeterol from 2.7 to $1.0 \mathrm{ng} \cdot \mathrm{mL}^{-1}$ was significant $(\mathrm{p}=0.02)$. The apparent elevation of MPO concentrations with addition of fluticasone from a mean of 3.2 to $5.2 \mathrm{ng} \cdot \mathrm{mL}^{-1}$ was not significant $(\mathrm{p}=0.4)$.

Relationships between inflammatory variables during the treatment period. For the whole group, changes in MPO level over time were significantly and strongly related to changes in IL-8 level $\left(\mathrm{r}^{2}=0.57, \mathrm{p}<0.001\right)$ (fig. 4$)$, and there was a weak but still significant relationship between changes in

Table 2. - Bronchoalveolar lavage fluid total cell and neutrophil counts and interleukin (IL)-8 and myeloperoxidase (MPO) levels before and after 12 weeks of additional inhaled fluticasone, salmeterol or placebo

\begin{tabular}{|c|c|c|c|c|c|c|}
\hline & \multicolumn{2}{|c|}{ Placebo } & \multicolumn{2}{|c|}{ Salmeterol } & \multicolumn{2}{|c|}{ Fluticasone } \\
\hline & Before & After & Before & After & Before & After \\
\hline Subjects $\mathrm{n}$ & \multicolumn{2}{|c|}{16} & \multicolumn{2}{|c|}{13} & \multicolumn{2}{|c|}{16} \\
\hline TCC $10^{3}$ cells $\cdot \mathrm{mL}^{-1}$ & $99(80-122)$ & $89(69-114)$ & $97(84-113)$ & $123(96-158)$ & $143(115-179)$ & $128(99-166)$ \\
\hline Neutrophils $10^{3}$ cells $\cdot \mathrm{mL}^{-1}$ & $1.9(1.3-2.9)$ & $2.4(1.4-4.0)$ & $2.2(1.4-3.4)$ & $1.7(1.1-2.6)$ & $2.2(1.1-4.4)$ & $4.6(2.6-7.9)^{* * *}$ \\
\hline $\mathrm{IL}-8 \mathrm{pg} \cdot \mathrm{mL}^{-1}$ & $63.6(17.9-167.3)$ & $61.2(6.0-265.8)$ & $72.8(16.1-308.8)$ & $61.2(8.3-186.1)^{\#}$ & $68.3(21.0-540.4)$ & $89.0(22.4-235.9)$ \\
\hline $\mathrm{MPO} \mathrm{ng} \cdot \mathrm{mL}^{-1}$ & $3.0(1.9-4.7)$ & $2.6(1.3-5.3)$ & $2.7(1.3-5.7)$ & $1.0(0.4-2.8)^{\top}$ & $3.2(1.8-6.0)$ & $5.2(3.3-8.1)$ \\
\hline
\end{tabular}

Data are presented as geometric mean (95\% confidence interval), and as median (range) for interleukin (IL)-8. TCC: total cell count; MPO: myeloperoxidase. ${ }^{* * *}: \mathrm{p}=0.001$ versus baseline; ${ }^{\#}: \mathrm{p}=0.06$ versus placebo, $\mathrm{p}=0.02$ versus baseline; ${ }^{\bullet}: \mathrm{p}=0.04$ versus $\mathrm{placebo}, \mathrm{p}=0.02$ versus baseline. 

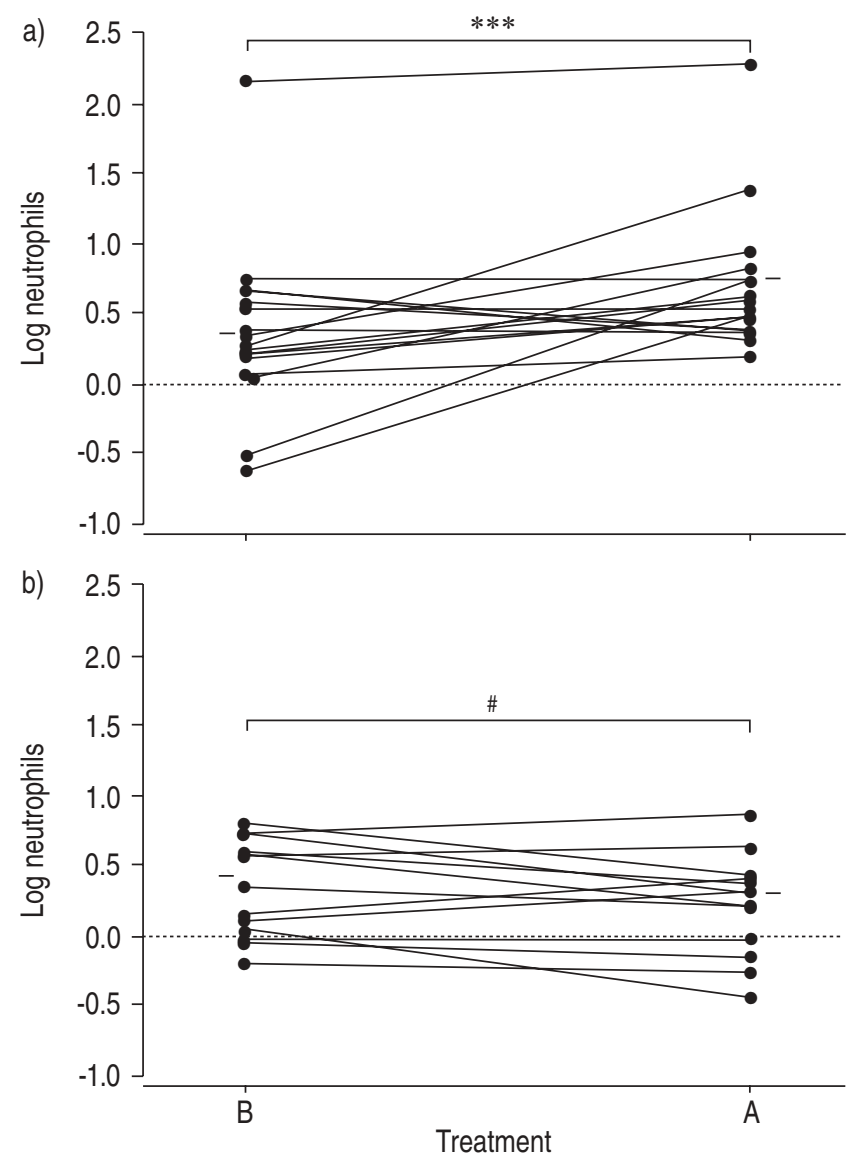

Fig. 1.-Bronchoalveolar lavage fluid neutrophil numbers before (B) and after (A) 12 weeks of: a) giving additional inhaled corticosteroid in the form of fluticasone; and b) introducing salmeterol. Horizontal bars represent means. Analysis of covariance indicated the changes with salmeterol and fluticasone were significantly different $(\mathrm{p}=0.007)$. Additionally, compared to baseline, the increase in neutrophil numbers with fluticasone was significant $(\mathrm{p}=0.001)$. ***: $\mathrm{p}=0.001$; \#: $\mathrm{p}=0.3$.

neutrophil number and IL-8 level $\left(\mathrm{r}^{2}=0.1, \mathrm{p}=0.03\right)$. Similarly, changes in MPO level were weakly but significantly related to changes in neutrophil number $\left(\mathrm{r}^{2}=0.17, \mathrm{p}=0.005\right)$ across all patients. Importantly, there was no relationship across treatment groups between changes in absolute number or percentage of neutrophils and eosinophils. Thus, the observed changes in BALF neutrophil number were not simply reflecting a general decrease in the absolute number or percentage of granulocytes. Similarly, there was no relationship between change in BALF eosinophil number and the observed changes in IL-8 and MPO level. Changes in PD20 and lung function with treatment were not related to any of the observed changes in IL-8, neutrophil or MPO level.

\section{Discussion}

The present study has demonstrated elevated levels of IL-8 in the BALF of subjects with persistent asthma despite treatment with low/moderate ICS doses. A strong relationship between IL-8 and MPO levels, but no significant relationship with airway neutrophil (or eosinophil) numbers, has also been demonstrated, which suggests IL-8 may function primarily as a neutrophil activator rather than a granulocyte chemoattractant in the present asthmatic population. It has been
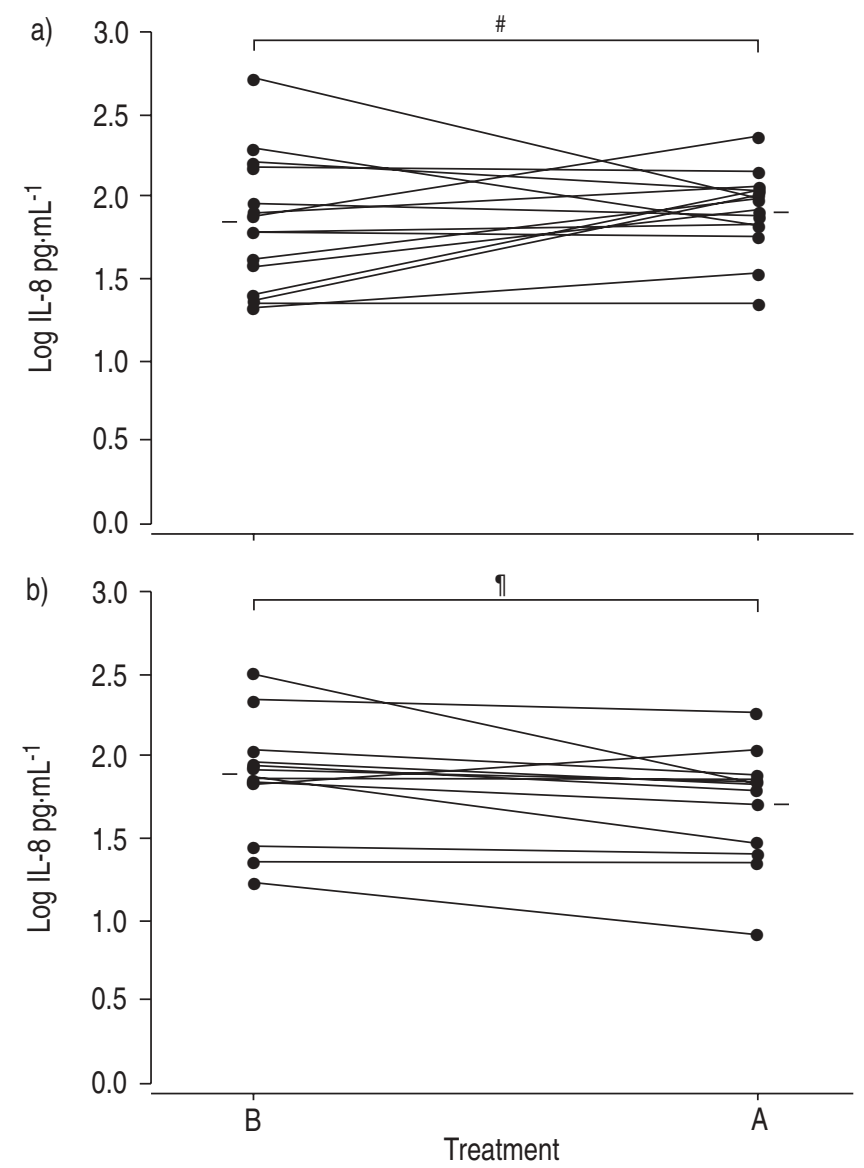

Fig. 2. - Bronchoalveolar lavage fluid interleukin (IL)-8 levels before (B) and after (A) 12 weeks of: a) giving additional inhaled corticosteroid in the form of fluticasone; and b) introducing salmeterol. Horizontal bars represent means. The Kruskal-Wallis test indicated the difference between changes with salmeterol and fluticasone almost reached significance $(\mathrm{p}=0.06)$. Additionally, there was a significant within-group reduction in IL-8 levels following salmeterol treatment $(\mathrm{p}=0.02){ }^{\#}: \mathrm{p}=0.3 ;{ }^{\uparrow}: \mathrm{p}=0.02$.

shown that introducing salmeterol in these subjects significantly reduces BALF IL-8 and MPO concentrations, suggesting a possible anti-inflammatory effect directed against IL-8 and neutrophil activation. Furthermore, adding salmeterol or alternatively increasing the ICS dose had significantly contrasting effects upon BALF neutrophil numbers and MPO levels and there was a strong trend towards divergent effects on IL-8 concentrations. Indeed, additional ICS in the form of fluticasone paradoxically increased BALF neutrophil numbers, suggesting a potentially pro-neutrophilic effect of higher-dose ICS.

Although previous reports have demonstrated increased IL-8 levels in the BALF of subjects with ICS-untreated asthma and in subjects with more severe disease, the present findings suggest that IL-8 may also play a role in persistent asthma treated with moderate ICS doses $[5,12,13]$. Previous findings, albeit in severe disease, of a significant relationship between IL-8 and MPO levels have also been confirmed, but no significant relationship between IL-8 levels and neutrophil numbers found [14]. These observations suggest that IL-8 may behave predominantly as a neutrophil activator in the stable asthma studied, although they leave the possibility of a more prominent role as a chemoattractant during exacerbations.

Interestingly, although a strong relationship between BALF IL-8 and MPO levels was demonstrated in the present 

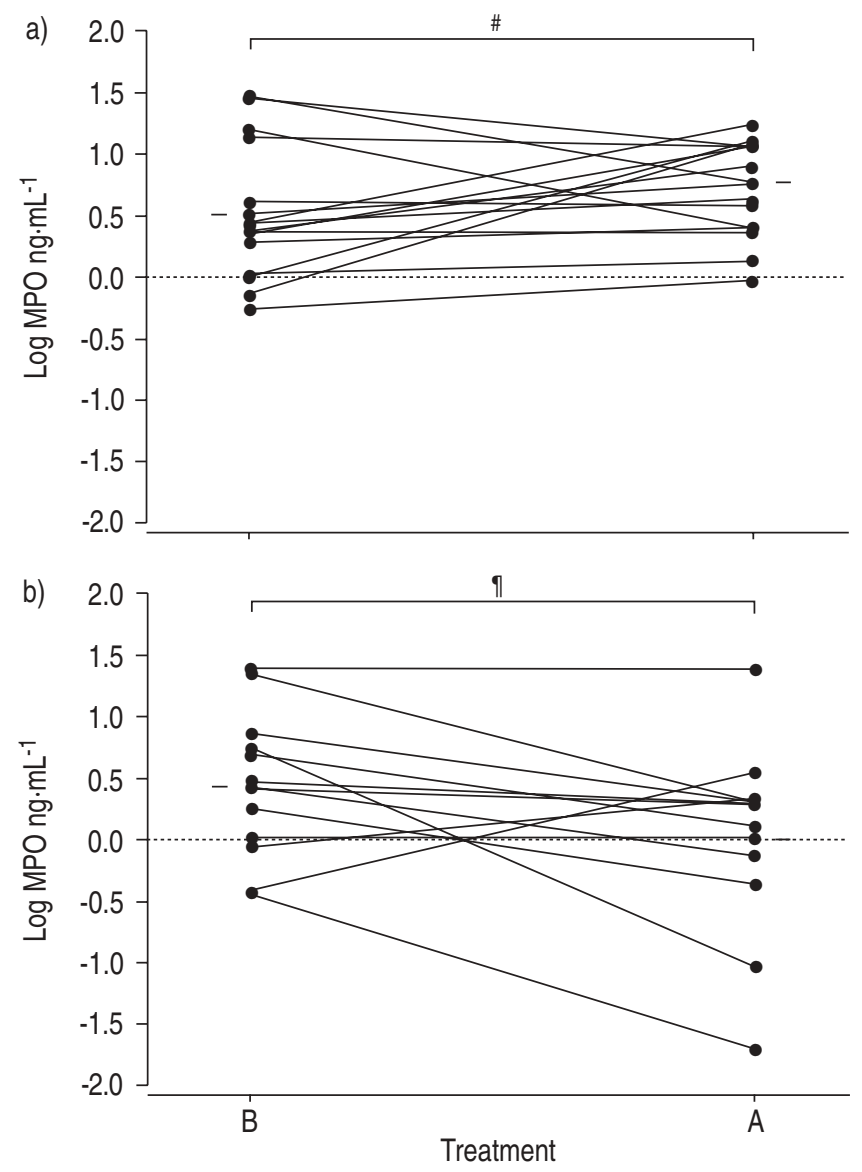

Fig. 3. - Bronchoalveolar lavage fluid myeloperoxidase (MPO) levels before (B) and after (A) 12 weeks of: a) giving additional inhaled corticosteroid in the form of fluticasone; and b) introducing salmeterol. Horizontal bars represent means. Analysis of covariance once more revealed significantly different effects between fluticasone and salmeterol $(\mathrm{p}=0.002)$, and changes with salmeterol were significant compared to placebo $(\mathrm{p}=0.04)$. Additionally, there was a significant within-group reduction in MPO levels following salmeterol treatment $(\mathrm{p}=0.02) .{ }^{\#}: \mathrm{p}=0.4 ;{ }^{\uparrow}: \mathrm{p}=0.02$.

asthmatic subjects, there was no significant overall difference in mean MPO levels between asthmatics and controls. Intuitively, it would be expected that MPO levels would increase with IL-8 levels, although endogenously occurring inhibitors of neutrophil activation such as secretory leukocyte protease inhibitor may have blunted this response. However, this explanation remains purely speculative [15] and assessment of MPO levels may not be a reliable or sensitive enough measure of neutrophil activity in the sort of stable asthma investigated in the present study.

Although treatment effects were small, there was a significant reduction in MPO levels compared to placebo and within-treatment group reduction in IL-8 levels with salmeterol, which strongly suggests some anti-inflammatory action. In-vitro studies suggest ICSs and LABAs may have synergistic anti-inflammatory effects directed against IL-8, perhaps mediated by complementary actions on nuclear transcription of the cytosolic glucocorticoid receptor [16-18]. These findings may be analogous to the reduction in MPO levels seen in the present study, if it is assumed that IL-8 is functioning predominantly as a neutrophil activator in this setting. Alternatively, salmeterol may directly inhibit the stimulated respiratory burst of airway neutrophils and thus reduce MPO concentrations. This effect may be mediated via either a $\beta_{2}$-receptor-dependent action or perhaps a $\beta_{2}$-receptorindependent mechanism, in which the action of naturally

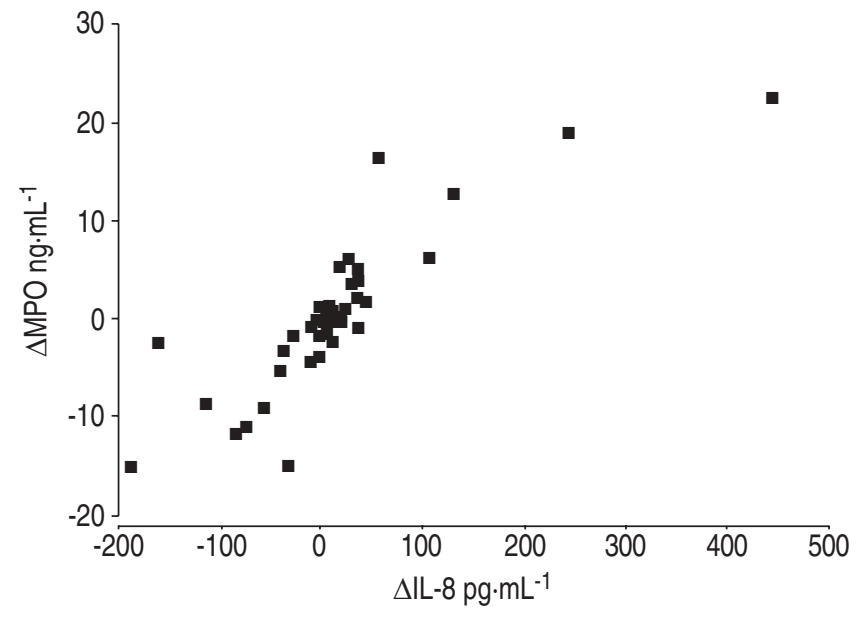

Fig. 4. - Relationship between changes $(\Delta)$ in bronchoalveolar lavage fluid myeloperoxidase (MPO) and interleukin (IL)-8 concentrations in asthmatic subjects across treatment groups $\left(r^{2}=0.57, p<0.0001\right)$.

occurring anti-inflammatory mediators such as IL-10 may be amplified [19]. The present data cannot address these issues but such potential mechanisms require further elucidation.

In contrast to salmeterol, increasing the effective ICS dose resulted in significantly elevated BALF neutrophil numbers but did not alter IL-8 or MPO levels. Although previous cross-sectional studies have suggested that high-dose systemic corticosteroids may promote airway neutrophilia, to the present authors' knowledge this is the first study to prospectively demonstrate an increase in airway neutrophil numbers following escalation of ICS dose. A reduction in neutrophil apopotosis or perhaps prolonged retention of neutrophils within the airway may explain the present findings, and, in this sense at least, ICSs may have a pro-inflammatory effect $[20,21]$.

In summary, the present findings support a possible pathogenic role for interleukin-8 in stable yet persistent asthma managed with moderate doses of inhaled corticosteroids. It may function predominantly as a neutrophilactivating cytokine in this population. Importantly, the current findings suggest a potential anti-inflammatory effect of salmeterol directed against interleukin-8 and possibly neutrophil activation, but, in contrast, it was also found that addition of higher doses of inhaled corticosteroids increased bronchoalveolar lavage fluid neutrophil numbers. The present observations reinforce current management guidelines in asthma, particularly with respect to the use of long-acting $\beta_{2}$-agonists in patients requiring moderate-dose inhaled corticosteroid therapy.

\section{References}

1. Djukanovic R, Roche WR, Wilson JW, et al. Mucosal inflammation in asthma. Am Rev Respir Dis 1990; 142: 434 457.

2. Kelly C, Ward C, Stenton CS, Bird G, Hendrick DJ, Walters EH. Number and activity of inflammatory cells in bronchoalveolar lavage fluid in asthma and their relation to airway responsiveness. Thorax 1988; 43: 684-692.

3. Sur S, Crotty TB, Kephart GM, et al. Sudden-onset fatal asthma. A distinct entity with few eosinophils and relatively more neutrophils in the airway submucosa? Am Rev Respir Dis 1993; 148: 713-719.

4. Wenzel SE, Szefler SJ, Leung DY, Sloan SI, Rex MD, Martin RJ. Bronchoscopic evaluation of severe asthma. 
Persistent inflammation associated with high dose glucocorticoids. Am J Respir Crit Care Med 1997; 156: 737-743.

5. Norzila MZ, Fakes K, Henry RL, Simpson J, Gibson PG. Interleukin-8 secretion and neutrophil recruitment accompanies induced sputum eosinophil activation in children with acute asthma. Am J Respir Crit Care Med 2000; 161: 769-774.

6. Ward C, Kelly CA, Stenton SC, Duddridge M, Hendrick DJ, Walters EH. The relative contributions of bronchoalveolar macrophages and neutrophils to lucigen- and luminol-amplified chemiluminescence. Eur Respir J 1990; 3: 1008-1014.

7. Barbato A, Panizzolo C, Gheno M, et al. Bronchoalveolar lavage in asthmatic children: evidence of neutrophil activation in mild-to-moderate persistent asthma. Pediatr Allergy Immunol 2001; 12: 73-77.

8. Gibson PG, Simpson JL, Saltos N. Heterogeneity of airway inflammation in persistent asthma: evidence of neutrophilic inflammation and increased sputum interleukin-8. Chest 2001; 119: 1329-1336.

9. Douwes J, Gibson P, Pekkanen J, Pearce N. Non-eosinophilic asthma: importance and possible mechanisms. Thorax 2002; 57: 643-648.

10. Anderson R, Feldman C, Theron AJ, Ramafi G, Cole PJ, Wilson R. Anti-inflammatory, membrane-stabilizing interactions of salmeterol with human neutrophils in vitro. $\mathrm{Br} \mathrm{J}$ Pharmacol 1996; 117: 1387-1394.

11. Li X, Ward C, Thien F, et al. An antiinflammatory effect of salmeterol, a long-acting $\beta_{2}$ agonist, assessed in airway biopsies and bronchoalveolar lavage in asthma. Am J Respir Crit Care Med 1999; 160: 1493-1499.

12. Xiu Q, Fujimura M, Nomura M, et al. Bronchial hyperresponsiveness and airway neutrophil accumulation induced by interleukin- 8 and the effect of the thromboxane $\mathrm{A}_{2}$ antagonist S-1452 in guinea-pigs. Clin Exp Allergy 1995; 25: $51-59$.
13. Nocker RE, Schoonbrood DF, van de Graaf EA, et al. Interleukin-8 in airway inflammation in patients with asthma and chronic obstructive pulmonary disease. Int Arch Allergy Immunol 1996; 109: 183-191.

14. Jatakanon A, Uasuf C, Maziak W, Lim S, Chung KF, Barnes PJ. Neutrophilic inflammation in severe persistent asthma. Am J Respir Crit Care Med 1999; 160: 1532-1539.

15. McElvaney NG, Nakamura H, Birrer P, et al. Modulation of airway inflammation in cystic fibrosis. In vivo suppression of interleukin-8 levels on the respiratory epithelial surface by aerosolization of recombinant secretory leukoprotease inhibitor. J Clin Invest 1992; 90: 1296-1301.

16. Korn SH, Jerre A, Brattsand R. Effects of formoterol and budesonide on GM-CSF and IL-8 secretion by triggered human bronchial epithelial cells. Eur Respir J 2001; 17: 10701077.

17. Pang L, Knox AJ. Synergistic inhibition by $\beta_{2}$-agonists and corticosteroids on tumor necrosis factor- $\alpha$-induced interleukin-8 release from cultured human airway smoothmuscle cells. Am J Respir Cell Mol Biol 2000; 23: 79-85.

18. Roth M, Johnson PRA, Rudiger JR, et al. Interaction between glucorticoids and $\beta_{2}$-agonists on bronchial airway smooth muscle cells through synchronised cellular signalling. Lancet 2002; 360: 1293-1299.

19. Ehrlich LC, Hu S, Peterson PK, Chao CC. IL-10 downregulates human microglial IL-8 by inhibition of NF- $\kappa \mathrm{B}$ activation. Neuroreport 1998; 9: 1723-1726.

20. Meagher LC, Cousin JM, Seckl JR, Haslett C. Opposing effects of glucocorticoids on the rate of apoptosis in neutrophilic and eosinophilic granulocytes. $J$ Immunol 1996; 156: 4422-4428.

21. Dale DC, Fauci AS, Wolff SM. Alternate-day prednisone. Leukocyte kinetics and susceptibility to infections. $N$ Engl J Med 1974; 291: 1154-1158. 